\title{
Therapeutic Hypothermia for Neonatal Encephalopathy
}

\section{Seetha Shankaran, MD}

\section{Opinion statement}

Neonatal Hypoxic-ischemic encephalopathy in full term infants has been associated with a high risk for morbidity and mortality. The patho-physiology of brain injury following hypoxiaischemia, noted in preclinical models, is a cascade of events resulting from excitotoxic and oxidative injury culminating in cell death. Hypothermia has been noted to be protective by inhibiting various events in the cascade of injury. Major randomized clinical trials in neonatal HIE have demonstrated reduction in death and disability and continued safety and efficacy of neuroprotection in childhood. There is now clinical and imaging evidence for hypothermia as neuroprotection. Hypothermia should be offered to term infants with either severe acidosis at birth or resuscitation needing continued ventilation and evidence of either moderate or severe encephalopathy within 6 hours of birth. The target temperature should be $33^{\circ}$ to $34^{\circ} \mathrm{C}$ and duration of cooling should be 72 hours, as per the published trials. Rewarming should be slow, at $0.5^{\circ} \mathrm{C}$ per hour. Infants should have serial neurological examinations during and at the end of cooling and at discharge. Multiorgan function should be supported and hypocarbia should be avoided during ventilator therapy. If available, the amplitude integrated EEG should be obtained prior to cooling and following rewarming. All infants should have magnetic resonance brain imaging studies within 1 to 2 weeks of age. Information from the neurological examination, aEEG and MRI studies will be helpful in discussing prognosis with parents. All infants should be followed for a minimum of 18 months to evaluate growth parameters and neurodevelopment al outcome.

\section{Keywords}

Therapeutic hypothermia; Neonatal encephalopathy; Term infants; Neonatal hypoxic-ischemic encephalopathy; Pathophysiology; Neurodevelopmental outcome; Neuroprotection; Head cooling; Whole body cooling; Randomized controlled trials; Knowledge gaps; Adjuvant therapies; Treatment

\section{Introduction}

Neonatal encephalopathy due to hypoxia-ischemia occurs in 1.5 (95\% CI 1.3 to 1.7 ) per 1000 live full term births $[1 \bullet \bullet$. Neonates with severe encephalopathy have a very high risk of death (up to $85 \%$ ) and an increased risk of cerebral palsy (CP) and mental retardation among survivors. Neonates with moderate encephalopathy have significant motor deficits, fine motor disability, memory impairment, visual or visuo-motor dysfunction, increased hyperactivity, and delayed school readiness $[2,3,4 \cdot \bullet, 5,6]$. The pathophysiology of brain injury secondary to hypoxic ischemia (HI) is associated with 2 phases; primary and secondary energy failure based on characteristics of the cerebral energy state documented in

\footnotetext{
(C) Springer Science+Business Media, LLC 2012

Address Neonatal-Perinatal Medicine, Children's Hospital of Michigan, \# 4C19, 3901 Beaubien Boulevard, Detroit, MI 48201, USA sshankar@med.wayne.edu.

Disclosure No potential conflicts of interest relevant to this article were reported.
} 
both preclinical models and human infants [7-10]. Primary energy failure is characterized by reductions in cerebral blood flow and oxygen substrates. High-energy phosphorylated compounds such as ATP and phosphocreatine are reduced and tissue acidosis is prominent. Primary energy failure is associated with an "excitotoxic-oxidative cascade" $[11,12 \bullet \cdot]$ with excessive stimulation of neurotransmitter receptors and membrane depolarization which mediates an increase in intracellular calcium and osmotic dysregulation [13•]. Intracellular calcium activates neuronal nitric oxide synthase leading to release of the oxygen free radical nitric oxide which can disrupt mitochondrial respiration. Signals released from damaged mitochondria lead to apoptosis or programmed cell death as long as energy supplies persist, but exhaustion of these supplies leads to cell necrosis. Apoptosis can also be triggered by activation of caspase enzymes. Resolution of hypoxia-ischemia within a specific time interval reverses the fall in high energy phosphorylated metabolites and intracellular $\mathrm{pH}$ and promotes recycling of neurotransmitters. If the injury is severe, the cascade of events results in a second interval of energy failure in the mitochondria, in which the brains energy supplies fall over a period of 24 hours [14*0]. Secondary energy failure differs from primary energy failure in that the declines in phosphocreatine and ATP are not accompanied by brain acidosis [9]. The pathogenesis of secondary energy failure involves continuation of the excitotoxic-oxidation cascade, apoptosis, inflammation and altered growth factors, and protein synthesis $[12 \bullet \bullet]$.

The interval between primary and secondary energy failure represents a "latent phase" that corresponds to a "therapeutic window." The duration of the window was noted to be approximately 6 hours in near-term fetal sheep treated with hypothermia initiated at varying intervals following timed HI injury $[15,16]$. Subsequent research has noted that cell death in the brain exposed to $\mathrm{HI}$ is delayed over several days to weeks after an injury and apoptosis and necrosis continue depending on the region and severity of the injury [12••].

Six large randomized controlled trials (RCT) of hypothermia for neonatal HIE have been published, all enrolling infants $\geq 36$ weeks or $\geq 35$ weeks gestation, within the therapeutic window of 6 hours, with severe acidosis or need for resuscitation at birth, and the presence of moderate or severe encephalopathy. These trials have demonstrated that either head cooling $[17,18 \bullet]$ or whole body cooling $[19,20 \bullet \bullet, 21 \bullet, 22 \bullet \bullet]$ decreases death or disability either in the entire cohort $[18 \bullet, 19,21 \bullet, 22 \bullet \bullet]$ or within subgroups of infants $[17,20 \bullet \bullet]$ at 18 to 24 months of age. Recent meta-analyses have demonstrated that hypothermia is effective and safe $[23 \bullet, 24 \bullet \cdot]$. Neuroprotection by hypothermia has now been demonstrated by magnetic resonance imaging (MRI) of the brain [25••, 26••].

\section{Treatment}

Prior to introduction of neuroprotection therapies, the management of neonates with HIE was limited to supportive intensive care. This included resuscitation in the delivery room followed by stabilization of hemodynamic and pulmonary dysfunction (hypotension, metabolic acidosis, cardiac dysfunction, and hypoventilation), correction of metabolic disturbances, (glucose [27••], calcium, magnesium, and electrolytes), treatment of seizures, and monitoring for multiorgan dysfunction.

\section{Method of cooling, duration, and depth of cooling}

Head cooling-The CoolCap study involved 234 term infants with moderate or severe encephalopathy and abnormal amplitude integrated EEG (aEEG), either cooledto a rectal temperature of $34^{\circ}$ to $35^{\circ} \mathrm{C}$ for 72 hours or conventional care. Primary outcome was death or severe disability at 18 months [17]. Data was unavailable for 16 infants (7\%). Death or disability occurred in $66 \%$ conventional care and $55 \%$ cooled group, adjusted OR (95\% CI) 0.61 (0.34-1.09) $P=0.10$. Predefined subgroup analysis suggested head cooling had no 
effect in infants with the most severe aEEG changes, but was beneficial in infants with less severe aEEG changes.

Selective head cooling or usual care was evaluated in an RCT in China where 256 infants were enrolled with encephalopathy [18•]. The cooling group was maintained at $34{ }^{\circ} \mathrm{C}$ nasopharyngeal and rectal temperature for 72 hours. Primary outcome was death or severe disability at 18 months. There were 21 post-randomization exclusions and of the remainder, $21 \%$ and $19 \%$ had mild encephalopathy in the hypothermia and usual care groups, respectively. Data was unavailable for 41 infants (16\% of the analyzed or $24 \%$ of the recruited cohort). The primary outcome occurred in $49 \%$ control and $31 \%$ hypothermia group infants, OR $0.47(0.26-0.84, P=0.01)$.

Whole body cooling-The NICHD RCT of whole body hypothermia for neonates with HIE [19] was preceded by an animal study of feasibility and a pilot study. Term infants ( $n=208$ ) with moderate or severe encephalopathy were randomly assigned to whole body cooling to an esophageal temperature of $33.5^{\circ}$ for 72 hours or usual care. Primary outcome was death or moderate/severe disability at 18 months. Data was unavailable for 3 infants (1 $\%$ ). Death or disability occurred in $62 \%$ usual care and $44 \%$ hypothermia group, RR (5\% CI) $0.72(0.54-0.95) P=0.01$.

The Total Body Hypothermia for Neonatal Encephalopathy (TOBY) trial [20••] enrolled 325 infants with moderate or severe encephalopathy and an abnormal aEEG. Infants were randomly assigned to whole body hypothermia to a rectal temperature of $33^{\circ}$ to $34{ }^{\circ} \mathrm{C}$ for 72 hours or usual care. Primary outcome was death or severe neurodevelopmental disability at 18 months. Data was unavailable for two infants $(<1 \%)$. Death or severe disability occurred in $53 \%$ usual care and $45 \%$ hypothermia group; RR $0.86(0.68-1.07) P=0.17$. Predefined secondary outcomes noted survival without disabilities was significantly higher in the cooled group compared with the usual care group. The rate of CP was lower and improved Mental and Psychomotor Indices were noted in the hypothermia group compared with the usual care group, all $P<0.05)$.

The European Network RCT [21•] enrolled 129 infants with moderate or severe encephalopathy and an abnormal aEEG. In the hypothermia (whole body) group, a rectal temperature of $33-34.0^{\circ} \mathrm{C}$ was maintained. All infants received morphine $(0.1 \mathrm{mg} / 1 \mathrm{~g})$ infusions. Primary outcome was death or disability at 18 months. Data on 18 infants (14\%) was not available. Death or severe disability occurred in $51 \%$ of the hypothermia group and $83 \%$ in the normothermia group, OR 21(0.09-0.54), $P=0.001$.

The most recent RCT published is the Infant Cooling Evaluation (ICE) trial [22••] ( $n=221)$. Whole body cooling was initiated at the referral hospital after clinical diagnosis of encephalopathy and rectal temperatures were monitored. Primary outcome was death or major disability at 24 months. Mild encephalopathy was noted in $15 \%$ hypothermia and 23 $\%$ control infants. Data was unavailable for $13(6 \%)$ of infants. The primary outcome occurred in $51 \%$ hypothermia and $66 \%$ control infants, RR $0.77(0.62-0.98)$. The mortality rate was significantly reduced while survival free of disability was increased in the hypothermia group compared with control group.

In summary, eligibility criteria for application of cooling should include severe acidosis and the presence of moderate or severe encephalopathy. The presence of encephalopathy has been shown to correlate with outcome; if the aEEG is used as additional eligibility criteria, it should be performed and interpreted by experienced individuals. Infants with moderate encephalopathy benefit from hypothermia therapy; based on recent meta-analyses, it is apparent that infants with severe encephalopathy also benefit from hypothermia. The depth 
of temperature should be $33^{\circ}$ to $34{ }^{\circ} \mathrm{C}$ and the duration of cooling should be 72 hours followed by rewarming at a rate of $0.5^{\circ} \mathrm{C}$ per hour.

\section{Predictors of outcome helpful to the clinician}

The neurological examination and presence of seizures at $<6$ hours during cooling or at discharge-A scoring system using clinical and laboratory values at $<6$ hours of age developed within the NICHD trial of whole body hypothermia revealed correct classification rates for death or disability at 18 months $(78 \%)$ and death alone $(71 \%)$. Severe encephalopathy predicted death or disability at $67 \%$ and $73 \%$ respectively, while classification and regression tree models had correct classification rates of $80 \%$ and $77 \%$, respectively [28]. Severe HIE (at $<6$ hours of age) and an abnormal aEEG (at $<9$ hours of age) were related to death or disability at 18 months in univariate analysis in another report; whereas severe HIE alone was predictive of outcome in the multivariate analysis [29••]. The importance of serial neurological examinations during the study intervention period is highlighted in a study demonstrating that at 24 and 48 hours, infants in the hypothermia group had less severe encephalopathy than infants in the control group. Persistence of severe encephalopathy at 72 hours increased the risk of death and disability after controlling for treatment group. The discharge exam improved the predictive value of the stage of HIE at $<6$ hours for death and disability [30••]. None of the trials to date have an adequate sample size to examine the impact of hypothermia separately on moderate and severe encephalopathy; however, a consistent trend in the decrease in frequency of disability in both moderate and severe encephalopathy was noted in the NICHD trial in the hypothermia group compared with the control group [31].

The CoolCap trial has also reported better outcomes with treatment with hypothermia and lower encephalopathy grade at random assignment and absence of seizures [32]. Infants with persistent moderate encephalopathy on day 4 had a more favorable prognosis compared with standard care [33]. Clinical seizures and severe encephalopathy at random assignment in the NICHD trial were noted to be associated with death or disability or mental development index <70 at 18 months of age in the univariate analysis; when controlling for treatment and stage of encephalopathy, clinical seizures no longer had an independent effect on outcome $[34 \cdot]$.

An important caveat to interpret our results is that clinical seizures, may well underestimate the frequency of EEG documented seizure activity. In summary, the neurological examination is important for characterizing the stage of encephalopathy at initiation of cooling and is an important biomarker of outcome.

Role of aEEG-In the NICHD trial, the addition of the aEEG pattern to HIE stage did not add to the predictive value of model of death or disability at 18 months; the area under the curve changed from 0.72 to $0.75(P=0.19)$ [29••]. The CoolCap trial noted that greater amplitude of the aEEG at $<6$ hours of age was associated with better outcome [32]. The TOBY trial has noted that more infants with severely abnormal aEEG died or had severe disability at 18 months $[20 \bullet \bullet$.

Time to initiate cooling and outcome at $\mathbf{1 8}$ to $\mathbf{2 4}$ months-Both the NICHD trial and the TOBY trial have reported that time to randomization and time to initiate cooling did not have an impact on outcome at 18 months [20••, 31]. Therefore it is important to stabilize a term neonate with acidosis/birth resuscitation and confirm the presence of encephalopathy within the 6 hour window prior to initiating hypothermia therapy. 
Ventilation during hypothermia therapy-In a secondary analysis evaluating early blood gas perimeters' and ventilation with outcome among NICHD trial participants, both minimum $\mathrm{PCO}_{2}$ and cumulative $\mathrm{PCO}_{2}(<35 \mathrm{~mm} \mathrm{Hg})$ at $<12$ hours of age were associated with increased risk of death or disability at 18 months [35••]. Overventilation and hypocarbia should be avoided when treating infants with HIE.

Avoidance of elevated temperature-Two of the trials have noted that elevated temperatures (among control group infants) were associated with an increased risk of death and disability $[32,36]$. A neutral thermal environment should be maintained until cooling is initiated.

\section{Risk of decreases in temperature below target during induction and} maintenance of cooling-Among cooled infants, infants who were at lower birth weight and those with a greater need for blood pressure support were reported in the NICHD trial to be at risk for decreases in temperature to $<32.0^{\circ} \mathrm{C}$ both during induction and maintenance of cooling [37••]. Other medications (anticonvulsants, sedatives, or neuromuscular blocking agents) were not associated with these temperatures decreases. However, phenobarbital administered prior to cooling in the NICHD trial resulted in lower target esophageal temperatures during the induction phase [38•]. The CoolCap study has noted that there is an interaction between hypothermia and birth weight, infants with lower weight had better outcomes with cooling [32]. Therefore, smaller, sicker neonates require very close monitoring during hypothermia therapy and may require additional therapy for blood pressure support.

Safety of hypothermia-The safety of hypothermia as neuro protection has been reported in the trials to date $[17,18 \bullet, 19,20 \bullet \bullet, 21 \bullet, 22 \bullet \bullet, 31]$. No increase in persistent pulmonary hypertension, use of inhaled nitric oxide, or need for extra corporeal membrane oxygenation has been noted among cooled infants compared with infants receiving standard of care. No increases in metabolic disturbances or cardiac arrhythmia have been reported [31]. Hypothermia is currently being recommended by health care policy makers (American Heart Association Guidelines for Cardiopulmonary Resuscitation and Emergency Cardiovascular Care, and the International Consensus on Cardiopulmonary Resuscitation, and Emergency Cardiovascular Care Science with Treatment Recommendations) stating that following resuscitation at birth among $\geq 36$ week gestation neonates with evolving moderate or severe encephalopathy, hypothermia should be offered in the context of clearly defined protocols similar to published trials $[39 \bullet \bullet, 40 \bullet \cdot$. Therefore, infants born depressed following severe acidosis at birth should be resuscitated in the delivery room and stabilized prior to evaluation and confirmation of moderate or severe encephalopathy before initiating hypothermia therapy.

The rewarming phase-Rewarming should be performed slowly, at $0.5^{\circ} \mathrm{C}$ per hour. Seizures during the rewarming phase have been reported. In addition, infants may develop hypotension, possibly due to vasodilatation during rewarming, so additional blood pressure support, and or volume support may be required during this phase. Smaller, sicker infants may require a greater period than 6 to 8 hours to achieve normothermia.

Pharmacokinetic (PK) of medications during hypothermia-Pharmacokinetic parameters of medications can be altered during hypothermia therapy [41•]. Morphine levels have been noted to be increased [42]. Gentamicin levels are not affected during cooling [43]. Phenobarbital and Topiramate levels accumulate during hypothermia therapy [44•]. The effects of hypothermia on drug metabolism, clearance, and response have been reviewed 
recently [45•]. Clinicians should monitor pharmacokinetic parameters as the risk of multiorgan injury may further impact drug metabolism and clearance.

\section{Brain imaging evidence of neuroprotection}

The TOBY trial evaluated neonatal magnetic resonance images (MRI) from 131 of 325 (40

$\%$ ) of trial participants [25••]. Hypothermia was associated with a reduction in lesions in the basal ganglia or thalamus, OR $0.36(0.15-0.84, P=0.02)$, white matter, OR $0.30(0.12-0.77$, $P=0.01)$ and abnormal posterior limb of the internal capsule, $(0.38,0.17-0.85, P=0.02)$. Cooled infants had more normal scans and fewer scans that were predictive of 18 month neuro motor abnormalities. The accuracy of prediction of death and disability by MRI was $0.84(0.74-0.94)$ in the cooled and $0.81(0.71-0.91)$ in the control group.

The NICHD NRN examined neonatal MRI scans on 136 of 208 (65\%) of trial participants [26••]. The infants were characterized based upon the pattern of injury on the MRI findings. Normal scans were noted among $52 \%$ hypothermia and $35 \%$ control infants $(P=0.06)$. Infants in the hypothermia group had fewer areas of infarction (12\%) compared with control group infants (22\%) $P=0.02$, anterior limb of the internal capsule abnormality $(P=0.05)$, and posterior limb of internal capsule injury ( $P=0.06)$. The MRI brain injury pattern correlated with outcome of death or disability and with disability in survivors at 18 months. Each point increase in the severity of the pattern of brain injury was independently associated with a two-fold increase in the odds of death or disability. The age of obtaining MRI scans is between 1 and 2 weeks of age.

\section{Childhood outcomes after hypothermia}

Six- to 8-year outcomes after hypothermia for neonatal encephalopathy-The CoolCap study investigators evaluated whether 18-22 months outcomes predicted functional outcomes at 7-8 years among 62 of 135 surviving children from the trial [46•]. Measures of self-care, mobility, and cognitive function were assessed by the WeeFIM parent questionnaire. Functional outcome at 7-8 years was associated with the 18 month assessments; this was noted among children with favorable and adverse outcomes.

The NICHD Neonatal Research Network trial evaluated cognitive, attention, and executive and visuospatial function, neurologic outcomes, and physical and psychosocial health, with data available among 190 of 208 trial participants [47••]. Primary outcome of death or IQ<70 was noted among $47 \%$ hypothermia and $62 \%$ control group children $(P=0.06)$. Secondary outcomes included the mortality rate of $28 \%$ and $44 \%(P=0.04)$, death or CP of $41 \%$ and $60 \%(P=0.02)$ and death and severe disability of $41 \%$ and $60 \%(P=0.03)$ in the hypothermia and control groups, respectively. These data extend support for the use of hypothermia for neonatal HIE.

\section{Knowledge gaps in hypothermia therapy}

During discussions with clinicians on the use of hypothermia for neonatal encephalopathy, the use of hypothermia without evidenced based data has been noted (Table 1).

Cooling during transport-Hypothermia administered during transport of high risk infants with possible or established encephalopathy has been associated with over cooling [48]. This is noted when cooling is initiated by methods that do not allow management of target temperature (ie, use of gel packs or ice packs), or when core temperature is not monitored continuously. Infants with severe encephalopathy are at greater risk for overcooling. The risk of rapid rewarming of an overcooled infant (with core temperatures below $32^{\circ} \mathrm{C}$ ) to the target temperature is unknown. The benefit of cooling during transport has not been demonstrated; earlier time to cooling has not been shown to impact outcome 
$[20 \bullet \bullet, 31]$. Hypothermia during transport of high risk infants should only be conducted with targeted core temperature monitoring; servo-controlled devices may soon be are available [49].

Cooling of preterm infants-There is currently no evidenced based data on benefits of cooling for hypoxic-ischemia for infants $₫ 6$ weeks gestation age; this is an area that needs to be investigated [50••]. One RCT is on-going evaluating hypothermia as neuroprotection for late preterm infants (NCT00620711).

Cooling of infants presenting $>\mathbf{6}$ hours of age-There is evidence of ongoing brain injury beyond the 6-hour therapeutic window; hence hypothermia as therapy for infants with moderate/severe HIE who present after 6 hours of birth is being evaluated (NCT0061477).

\section{Future of hypothermia}

The rate of death or disability following hypothermia at 33 to $34{ }^{\circ} \mathrm{C}$ for 72 hours for neonatal HIE is unacceptably high and ranges from $31 \%$ to $55 \%$ in the published trials. Hence, optimizing cooling strategies further or adjuvant therapies are needed. Preclinical studies have noted that cooling to a depth of $5{ }^{\circ} \mathrm{C}$ may be more protective than cooling to a depth of $3{ }^{\circ} \mathrm{C}$. In addition, since injury is ongoing, a duration of cooling for $>72$ hours may be more protective that cooling for 72 hours. The NICHD is currently evaluating an optimizing cooling strategy by examining a greater depth of cooling $\left(32.0^{\circ} \mathrm{C}\right)$ and longer duration of cooling (120 hours) to decrease death and disability at 18 months (NCT01192776).

Hypothermia plus adjuvant therapies-Hypothermia plus adjuvant therapies have

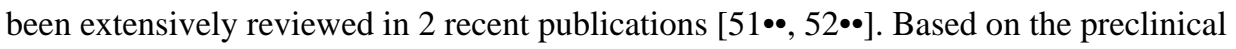
studies, ongoing trials in neonates include: inhaled Xenon and cooling (NCT01545271 and NCT00934700), safety of Erythropoietin (NCT00719407), Darbepoetin and hypothermia (NCT0147105), and Topiramate plus hypothermia (NCT01241019). The use of human cord blood for the hypoxic-ischemic neonate is also being investigated. A recent review highlights the approaches to this therapy [53••].

In summary, hypothermia for neonatal HIE is an effective therapy with a number needed to treat (NNT) of only 6 infants. The safety of hypothermia has been clearly demonstrated; however, clinical application must be monitored closely (Table 2). The neuroprotective effects noted at 18 months of age persist to childhood. The future of optimizing hypothermia therapy with additional adjuvant agents to reduce death or disability to a greater extent than current hypothermia therapy alone holds promise.

\section{Acknowledgments}

Dr. Shankaran has received grant support from the National Institute of Child Health \& Human Development.

\section{References and Recommended Reading}

Papers of particular interest, published recently, have been highlighted as:

- Of importance

•- Of major importance

1••. Kurinczuk JJ, White-Koning M, Badawi N. Epidemiology of neonatal encephalopathy and hypoxic-ischemic encephalopathy. Early Hum Dev. 2010; 86:329-38. [PubMed: 20554402] This article reviews the epidemiology of encephalopathy due to all causes. 
2. Shankaran S, Woldt E, Koepke T, et al. Acute neonatal morbidity and long-term central nervous system sequelae of perinatal asphyxia in term infants. Early Hum Dev. 1991; 25:135-48. [PubMed: 1713544]

3. Robertson, CMT. Long-term follow-up of term infants with perinatal asphyxia. In: Stevenson, DK.; Benitz, WE.; Sunshine, P., editors. Fetal and neonatal brain injury. 3rd ed. Cambridge University; New York: 2003. p. 829-58.

4••. De Vries LS, Jongmans MJ. Long-term outcome after neonatal hypoxic-ischemic encephalopathy. Arch Dis Child Fetal Neonatal Ed. 2010; 95:F220-4. [PubMed: 20444814] Excellent article on outcomes in childhood following neonatal hypoxic-ischemic encephalopathy.

5. Marlow N, Rose AS, Rands CE, et al. Neuropsychological and educational problems at school age associated with neonatal encephalopathy. Arch Dis Child Fetal Neonatal Ed. 2005; 90:F380-7. [PubMed: 16113154]

6. Gonzalez FF, Miller SP. Does perinatal asphyxia impair cognitive function without cerebral palsy? Arch Dis Child Fetal Neonatal Ed. 2006; 91:454-9.

7. Hope PL, Costello AM, Cady EB, et al. Cerebral energy metabolism studied with phosphorus NMR spectroscopy in normal and birth-asphyxiated infants. Lancet. 1984; 2:366-70. [PubMed: 6147452]

8. Azzopardi D, Wyatt JS, Cady EB, et al. Prognosis of newborn infants with hypoxic-ischemic brain injury assessed by phosphorus magnetic resonance spectroscopy. Pediatr Res. 1989; 25:445-51. [PubMed: 2717259]

9. Lorek A, Takei Y, Cady EB, et al. Delayed ("second-ary") cerebral energy failure after acute hypoxia-ischemia in the newborn piglet: continuous 48-hour studies by phosphorus magnetic resonance spectroscopy. Pediatr Res. 1994; 36:699-706. [PubMed: 7898977]

10. Blumberg RM, Cady EB, Wigglesworth JS, et al. Relation between delayed impairment of cerebral energy metabolism and infarction following transient focal hypoxia-ischemia in the developing brain. Exp Brain Res. 1997; 113:130-7. [PubMed: 9028781]

11. Johnston MV, Trescher WH, Ishida A, et al. Neurobiology of hypoxic-ischemic injury in the developing brain. Pediatr Res. 2001; 49:735-41. [PubMed: 11385130]

12••. Johnston MV, Fatemi A, Wilson MA, Northington F. Treatment advances in neonatal neuroprotection and neurointensive care. Lancet Neurol. 2011; 10:372-82. [PubMed: 21435600] Excellent and thorough overview of the pathophysiology of hypoxia-ischemic in the preclinical model and among human infants.

13. Cross JL, Meloni BP, Bakker AJ, et al. Modes of neuronal calcium entry and homeostasis following cerebral ischemia. Stroke Res Treat. 2010; 316862:1-9. Pathophysiology of one of the cascade of deleterious events that occurs following timed hypoxic-ischemic injury.

14••. Hagberg H, Mallard C, Rousset CI, et al. Apoptotic mechanisms in the immature brain: involvement of mitochondria. J Child Neurol. 2009; 24:1141-6. [PubMed: 19574577] This article will further our understanding of how the cell responds when hypoxia-ischemia occurs.

15. Gunn AJ, Gunn TR, de Haan HH, et al. Dramatic neuronal rescue with prolonged selective head cooling after ischemia in fetal lambs. J Clin Invest. 1997; 99:248-56. [PubMed: 9005993]

16. Gunn AJ, Bennet L, Gunning MI, et al. Cerebral hypothermia is not neuroprotective when started after postischemic seizures in fetal sheep. Pediatr Res. 1999; 46(3):274-80. [PubMed: 10473041]

17. Gluckman PD, Wyatt J, Azzopardi DV, et al. Selective head cooling with mild systemic hypothermia after neonatal encephalopathy: multicenter randomized trial. Lancet. 2005; 365:66370. [PubMed: 15721471]

18•. Zhou WH, Cheng GQ, Shao XM, et al. Selective head cooling with mild systemic hypothermia after neonatal hypoxic-ischemic encephalopathy: a multicenter randomized controlled trial in China. J Pediatr. 2010; 157:367-72. 372.e1-3. [PubMed: 20488453] RCT of head cooling for neonatal HIE in a middle income resourced country.

19. Shankaran S, Laptook AR, Ehrenkranz RA, et al. Whole-body hypothermia for neonates with hypoxic-ischemic encephalopathy. N Engl J Med. 2005; 353:1574-84. [PubMed: 16221780]

20••. Azzopardi DV, Strohm B, Edwards AD, et al. Moderate hypothermia to treat perinatal asphyxial encephalopathy. N Engl J Med. 2009; 361:1349-58. [PubMed: 19797281] Largest trial of whole body cooling for neonatal HIE, very well controlled, and excellent follow rates. Largest trial of whole body cooling for neonatal HIE, very well controlled, and excellent follow rates. 
21 . Simbruner G, Mittal RA, Rohlmann F, et al. Systemic hypothermia after neonatal encephalopathy: outcomes of neo.nEURO.network RCT. Pediatrics. 2010; 126(4):e771-8. [PubMed: 20855387] Another recent RCT, the primary outcome demonstrated neuroprotection of hypothermia, limitations have been discussed.

22••. Jacobs SE, Morley CJ, Inder TE, et al. Whole-body hypothermia for term and near-term newborns with hypoxic-ischemic encephalopathy: a randomized controlled trial. Arch Pediatr Adolesc Med. 2011; 165(8):692-700. PMID: 21464374. [PubMed: 21464374] A very well done RCT of whole body hypothermia for neonatal HIE; included initiation of treatment prior to arrival at the cooling center due to long distances that needed to be covered.

23•. Tagin MA, Woolcott CG, Vincer MJ, et al. Hypothermia for neonatal hypoxic ischemic encephalopathy: an updated systematic review and meta-analysis. Arch Pediatr Adolesc Med. 2012; 166(6):558-66. [PubMed: 22312166] Excellent meta-analyses of trials performed for neuroprotection with hypothermia for neonatal HIE.

24••. Edwards AD, Brocklehurst P, Gunn AJ, et al. Neurological outcomes at 18 months of age after moderate hypothermia for perinatal hypoxic ischemic encephalopathy: synthesis and metaanalysis of trial data. BMJ. 2010; 9:340-c363. Excellent meta-analyses of trials for hypothermia for neuroprotection foe neonatal HIE.

25••. Rutherford M, Ramenghi LA, Edwards AD, et al. Assessment of brain tissue injury after moderate hypothermia in neonates with hypoxic-ischemic encephalopathy: a nested substudy of a randomized controlled trial. Lancet Neurol. 2010; 9:39-45. [PubMed: 19896902] Excellent paper demonstrates brain injury following hypothermia for neonatal HIE.

26••. Shankaran S, Barnes PD, Hintz SR, et al. Brain Injury Following Trial of hypothermia for neonatal hypox-ic-ischaemic encephalopathy. Arch Dis Child. 2012:1-9. doi:10.1136/ archdischild-2011-301524. [PubMed: 22082500] Excellent paper demonstrates brain injury following hypothermia for neonatal HIE.

27••. Tam EWY, Haeusslein LA, Bonafacio SL, et al. Hypoglycemia is associated with increased risk of brain injury and adverse neurodevelopmental outcome in neonates at risk for encephalopathy. J Pediatr. 2012; 161:88-93. [PubMed: 22306045] Very interesting study showing hypoglycemia increases risk of brain injury among at-risk term infants.

28. Ambalavanan N, Carlo WA, Shankaran S, et al. Predicting outcomes of neonates diagnosed with hypoxemic-ischemic encephalopathy. Pediatrics. 2006; 118(5):2084-93. [PubMed: 17079582]

29••. Shankaran S, Pappas A, McDonald SA, et al. Predictive value of an early amplitude integrated electro-encephalogram and neurologic examination. Pediatrics. 2011; 128:e112-20. [PubMed: 21669899] This paper adds to the growing body of data on role of aEEG as a predictor of outcome following an RCT of HIE for neuroprotection.

30••. Shankaran S, Laptook AR, Tyson JE, et al. Evolution of encephalopathy during whole body hypothermia for neonatal hypoxic-ischemic encephalopathy. J Pediatr. 2012; 160:567-72.e3. [PubMed: 22050871] This paper stresses the importance of standardized neurological examinations during the clinical course of treatment with hypothermia for neuroprotection.

31. Shankaran S, Pappas A, Laptook AR, et al. Outcomes of safety and effectiveness in a multicenter randomized, controlled trial of whole-body hypothermia for neonatal hypoxic-ischemic encephalopathy. Pediatrics. 2008; 122:e791-8. [PubMed: 18829776]

32. Wyatt JS, Gluckman PD, Liu PY, et al. Determinants of outcomes after head cooling for neonatal encephalopathy. Pediatrics. 2007; 119(5):912-21. [PubMed: 17473091]

33. Gunn AJ, Wyatt JS, Whitelaw A, et al. Therapeutic hypothermia changes the prognostic value of clinical evaluation of neonatal encephalopathy. J Pediatr. 2008; 152:55-8. 58.e1. Epub 2007 Oct 24. [PubMed: 18154900]

34•. Kwon JM, Guillet R, Shankaran S, et al. Clinical seizures in neonatal hypoxic-ischemic encephalopathy have no independent impact on neurodevelopmental outcome: secondary analyses of data from the neonatal research network hypothermia trial. J Child Neurol. 2011; 26(3):322-8. [PubMed: 20921569] The role of seizures in predicting outcome in neonates treated with hypothermia in an RCT for neuroprotection is assessed.

35••. Pappas A, Shankaran S, Laptook AR, et al. Hypocarbia and adverse outcome in neonatal hypoxic-ischemic encephalopathy. J Pediatr. 2011; 158:752-58.e1. [PubMed: 21146184] This 
paper evaluates the role of early ventilation and risk of hypocarbia that may impact outcome for neonates with HIE.

36. Laptook A, Tyson J, Shankaran S, et al. Elevated temperature after hypoxic-ischemic encephalopathy: A risk factor for adverse outcome. Pediatrics. 2008; 122:491-9. [PubMed: 18762517]

37••. Shankaran S, Laptook AR, McDonald SA, et al. Temperature profile and outcomes of neonates undergoing whole body hypothermia for neonatal hypoxic-ischemic encephalopathy. Pediatr Crit Care Med. 2012; 13(1):53-9. [PubMed: 21499182] This study helps further our understanding of how cooling is tolerated among infants with HIE.

38•. Sant'Anna G, Laptook AR, Shankaran S, et al. Phenobarbital and temperature profile during hypothermia for hypoxic-ischemic encephalopathy. J Child Neurol. 2012; 27(4):451-7. [PubMed: 21960671] This study helps the reader to understand how infants with HIE tolerate cooling when being treated with anticonvulsant phenobarbital.

39••. Kattwinkel J, Perlman JM, Aziz K, et al. Neonatal resuscitation: 2010 American Heart Association Guidelines for Cardiopulmonary Resuscitation and Emergency Cardiovascular Care. Pediatrics. 2010; 126(5):e1400-13. [PubMed: 20956432] This is a very important paper for all to read as guidelines are based on this document.

40••. Perlman JM, Wyllie J, Kattwinkel J, et al. Neonatal Resuscitation: 2010 International Consensus on Cardiopulmonary Resuscitation and Emergency Cardiovascular Care Science with Treatment Recommendations. Pediatrics. 2010; 126(5):e1319-44. [PubMed: 20956431] This is a very important paper for all to read as practice guidelines are based on this document.

41 . Zanelli S, Buck M, Fairchild K. Physiologic and pharmacologic considerations for hypothermia therapy in neonates. J Perinatol. 2011; 31(6):377-86. [PubMed: 21183927] This is an important review discussing a knowledge gap in treatment with hypothermia.

42. Róka A, Melinda KT, Vásárhelyi B, et al. Elevated morphine concentrations in neonates treated with morphine and prolonged hypothermia for hypoxic ischemic encephalopathy. Pediatrics. 2008; 121(4):e844-9. [PubMed: 18381513]

43. Liu X, Borooah M, Stone J, et al. Serum gentamicin concentrations in encephalopathic infants are not affected by therapeutic hypothermia. Pediatrics. 2009; 124(1):310-5. [PubMed: 19564314]

44- Filippi L, la Marca G, Cavallaro G, et al. Phenobarbital for neonatal seizures in hypoxic ischemic encephalopathy: a pharmacokinetic study during whole body hypothermia. Epilepsia. 2011; 52(4):794-801. [PubMed: 21371018] This paper sheds light on a knowledge gap in hypothermia therapy.

45•. van den Broek MP, Groenendaal F, Egberts AC, et al. Effects of hypothermia on pharmacokinetics and pharmacodynamics: a systematic review of preclinical and clinical studies. Clin Pharmacokinet. 2010; 49(5):277-94. [PubMed: 20384391] This is an important review discussing a knowledge gap in treatment with hypothermia.

46•. Guillet R, Edwards AD, Thoresen M, et al. Seven- to 8-year follow-up of the CoolCap trial of head cooling for neonatal encephalopathy. Pediatr Res. 2012; 71(2):205-9. [PubMed: 22258133] This paper discusses ability to predictive outcome at 7-8 years from the 18-24 month assessment of neonatal HIE treated with hypothermia.

47••. Shankaran S, Pappas A, McDonald SA, et al. Childhood outcomes after hypothermia for neonatal encephalopathy. N Engl J Med. 2012; 366:2085-92. [PubMed: 22646631] The first paper discussing childhood outcome after hypothermia for neonatal HIE, demonstrating continued support for its use for this indication.

48. Fairchild K, Sokora D, Scott J, et al. Therapeutic hypothermia on neonatal transport: 4-year experience in a single NICU. J Perinatol. 2010; 30(5):324-9. [PubMed: 19847186]

49. Kendall GS, Kapetanakis A, Ratnavel N, et al. Cooling on Retrieval Study Group. Passive cooling for initiation of therapeutic hypothermia in neonatal encephalopathy. Arch Dis Child Fetal Neonatal Ed. 2010; 95(6):F408-12. [PubMed: 20870910]

$50 \bullet$. Higgins RD, Raju T, Edwards AD, et al. Hypothermia and other treatment options for neonatal encephalopathy: an executive summary of the Eunice Kennedy Shriver NICHD workshop. J Pediatr. 2011; 159:851-8. [PubMed: 21875719] Very comprehensive state of the art document on hypothermia for neuroprotection for neonatal HIE. 
$51 \bullet$. Robertson NJ, Tan S, Groenendaal F, et al. Which neuroprotective agents are ready for bench to bedside translation in the newborn infant? J Pediatr. 2012; 160(4):544-52.e4. [PubMed: 22325255] Excellent review of the next phase of adjunctive therapy as neuroprotection for neonatal HIE.

52•. Cilio MR, Ferriero DM. Synergistic neuroprotective therapies with hypothermia. Semin Fetal Neonatal Med. 2010; 15(5):293-8. [PubMed: 20207600] An excellent review of future of neuroprotection therapies for neonatal HIE.

53••. Pimentel-Coelho PM, Rosado-de-Castro PH, da Fonseca LM, et al. Umbilical cord blood mononuclear cell transplantation for neonatal hypoxic-ischemic encephalopathy. Pediatr Res. 2012; 71(4 Part 2):464-73. [PubMed: 22430382] This paper discusses a very new therapy being investigated as neuroprotection. 

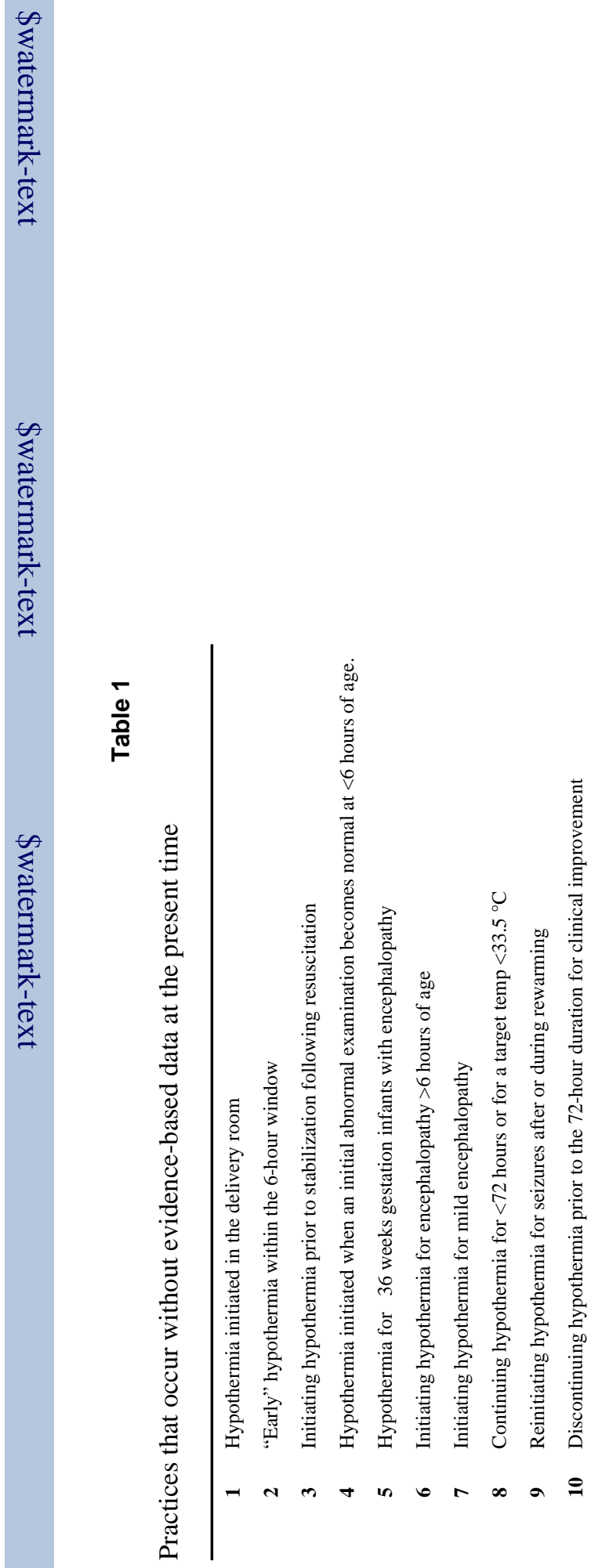

Curr Treat Options Neurol. Author manuscript; available in PMC 2013 December 01. 


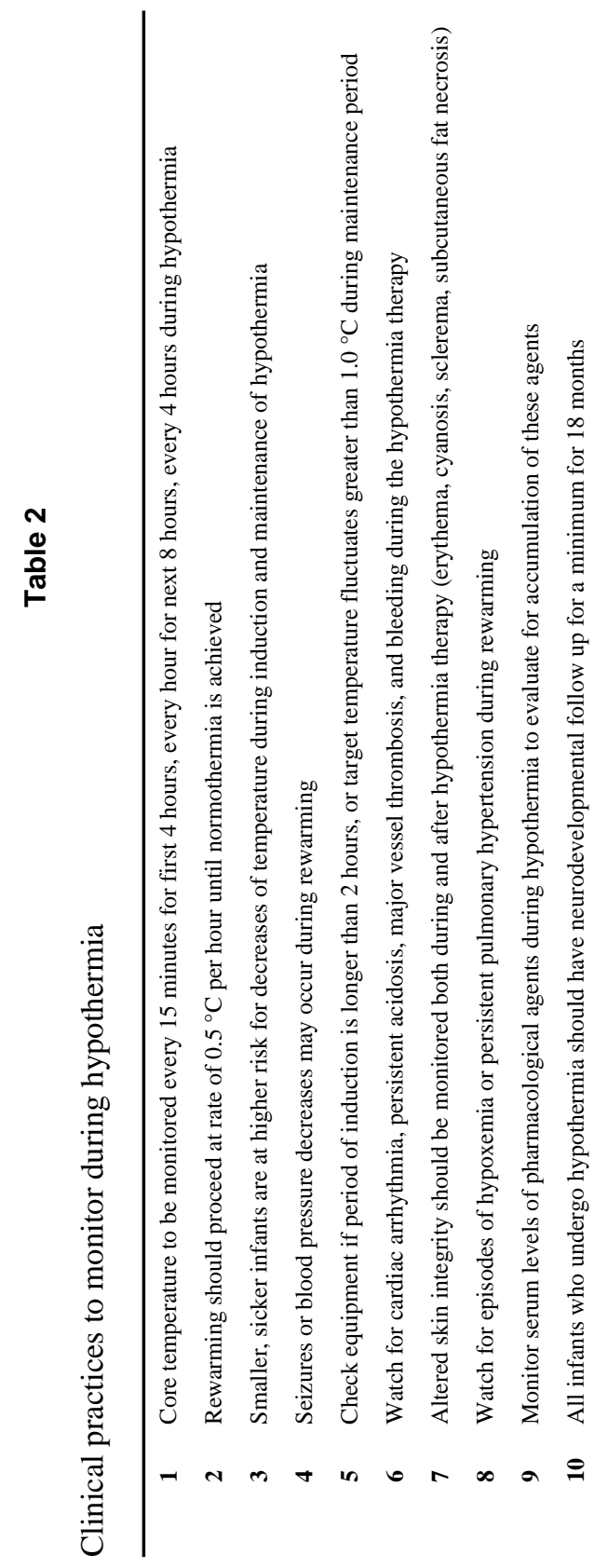

Curr Treat Options Neurol. Author manuscript; available in PMC 2013 December 01. 